\section{Measurement of Human Performance}

For many years psychologists have been interested in the specific and general causes leading to the observed diminution of efficiency in behaviour concerned with regular adaptation to a repeated stimulus. Certain generalizations seem to be valid ; for example, that after some time efficiency decreases, though not always regularly, and that various, apparently irrelevant, circumstances can affect the results. These studies have been limited, however, and no investigator has had a sufficiently large sample of subjects nor adequate controls. In the Special Report Series No. 268 of the Medical Research Council, "Researches on the Measurement of Human Per. formance", by N. H. Mackworth (pp. 156 ; London H.M. Stationery Office, 1950 ; 4s, net), the study reported had the advantage of being urgently required by the Armed Services, since during the Second World War there was much work involving prolonged visual search both with the naked eye and with the aid of radar screens. Obviously, continued accuracy was essential. A series of tests for visual and auditory vigilance was devised as nearly as possible reproducing the real situation, and the subjects were from the Services. The tests and the conditions involved are described in detail, and many of them might be adapted to the similar or related problems existing in other fields. Statistics are given showing the effect of different periods of duty, of knowledge of the results, of the effects of 'Benzedrine', of individual differences and of high atmospheric temperatures. The second part of the work gives the results of the study of environmental stress, for example, the effects of tear gas and of arsenical smoke, and further studies of high temperatures. The results are analysed in detail and the inter-relationship between physical, physiological and psychological findings clearly brought out. This scholarly and scientific report is a valuable contribution to knowledge both from the practical and theoretical points of view.

\section{Group Behaviour}

THE sixteenth issue of Science News contains an interesting article by G. R. Taylor on group dynamics. After reviewing recent work on the mechanics of group psychology, Taylor suggests that behaviour is not simply a product of personality, as has always been assumed, but is the product of interaction between personality and the culture of the group. The implications of this conclusion are far-reaching and mean that, if we wish to obtain higher output from industrial workers, we have to study not only individual motivation ('incentives') but also the dynamies of the work-teams and factory groups in which they find themselves. It is also suggested that there is a valid sense in which a group may be said to have a life -intentions, anxieties and patterns of behaviour-which is distinct from the life of the individuals composing it. The next stage in the study of group behaviour is an exhaustive analysis of a few representative groups in terms of all the known variables, to establish whether they are adequate to give a complete account of what goes on in the group. In another article D. W. Davies suggests that, given the necessary time for computation, games of chess and other two-person games can only lead to foregone conclusions as in noughts and crosses. Stanley Byard also gives an account of robots which play games, while Prof. H. Hartridge concludes his previous article on human colour vision. Besides other articles, there is the usual research report by Arthur Haslett.

\section{Use of Mechanical Power in Agriculture}

IN his address to the Farmers' Club at a meeting held in the Royal Empire Society's Hall, London, on November 6, Mr. W. H. Cashmore chose the subject of a "Survey of Power on the Land". After showing how rapid has been the advance in mechanization in Great Britain since 1939, Mr. Cashmore went on to discuss the general trends in this direction at the present day, emphasizing in particular the necessity for a reduction in working costs. Improvements have been made in the handling of hay, silage and grass for drying. One-man pick-up balers have already speeded up the collection of hay sixfold, and a recently introduced self-propelled machine can increase the rate still further, while the value of bruising machinery to hasten the natural drying process and so reduce the risk of damage to quality is being tested. Other new developments in grass conservation are one-man outfits for collecting silage material and automatic feeding devices for grass-drying plants. Although improvements in technique have lowered the average surplus moisture in grain harvested by combine, con. siderable attention still has to be paid to the subsequent drying processes, and two methods, the sack platform drier and the silo drying installation, have been established as satisfactory for this purpose. Machines for harvesting root crops are being more extensively used, and from trials carried out by the National Institute of Agricultural Engineering it seems that, as regards potato lifting, work should proceed with a two-row machine depositing the tubers on the ground as well as with complete harvesters. The needs of the small farm present a special difficulty, and versatile machines such as the universal root harvester are of particular value here. Though there are indications that a phase of stability in design is being reached, some new machines, such as one that combines drills and fertilizer placement, and a one-way plough, are still needed. The prospects of a decrease in capital outlay are not hopeful ; but if greater attention is paid to reliability and simplified maintenance and to the elimination of weak component parts, there is no doubt that working-costs can be materially reduced.

\section{Supply of High-Purity Metals in Great Britain}

ON several occasions, and particularly during the Second World War, important research projects in Great Britain have been delayed through lack of metals of suitable purity, and to obviate this in future the Department of Scientific and Industrial Research, through its Chemistry Research Board, set up a Pure Metals Committee. The task of this Committee has been to determine, by means of thorough analyses, which metals are actually aveilable in a high state of purity, and to promote the manufacture of batches of higher-purity material where this has been necessary. On the advice of the Committee, the Department has acquired limited quantities of some of these latter metals, and others are being obtained. The Department also has a considerable amount of information about these elements which will be published more fully in the near future. On the basis of this, the following classification into groups has been made : (1) metals available commercially in a purity approaching 